\title{
Fútbol sala y alto rendimiento: revisión sistemática de la literatura 2015-2020
}

\author{
High-performance and futsal: a systematic review of the literature 2015-2020
}

\author{
Alberto Sanmiguel-Rodríguez ${ }^{1-2}$, Sixto González-Víllora ${ }^{3}$, Víctor Arufe Giráldez ${ }^{4}$ \\ 1 Facultad de Lenguas y Educación. Universidad Camilo José Cela. España. \\ 2 Facultad de Lenguas y Educación. Universidad Antonio de Nebrija. España. \\ 3 Facultad de Educación. Universidad de Castilla-La Mancha. España. \\ 4 Facultad de Ciencias de la Educación. Universidad de A Coruña. España. \\ CORRESPONDENCIA: \\ Alberto Sanmiguel-Rodríguez \\ asrgz2014@gmail.com \\ Recepción: octubre 2020 • Aceptación: abril 2021 \\ CÓMO CITAR EL ARTÍCULO: \\ Sanmiguel-Rodríguez, A., González-Víllora, S., \& Arufe Giráldez, \\ V. (2021). Fútbol sala y alto rendimiento: revisión sistemática de \\ la literatura 2015-2020. Cultura, Ciencia y Deporte, 16(49), 465- \\ 476. http://dx.doi.org/10.12800/ccd.v16i49.1587
}

\section{Resumen}

El fútbol sala es un deporte con un importante número de practicantes. Su reducido campo de juego y la rapidez en las acciones de los jugadores anima a investigar sobre sus parámetros configuradores para conocer estrategias que mejoren el rendimiento deportivo. El objetivo de este trabajo es revisar los artículos publicados entre los años 2015 y 2020 que versen sobre fútbol sala de élite. Se realizó una búsqueda bibliográfica en las bases de datos Scopus y Dialnet utilizando diferentes combinaciones de los términos: "futsal", "fútbol sala", "élite", "profesional" y "alto rendimiento". Se encontraron un total de 114 artículos, analizándose 50 trabajos tras la aplicación de los criterios de inclusión. Se establecieron tres grandes categorías: 1 ) investigaciones relacionadas con condicionantes del juego, 2) investigaciones relacionadas con cargas de entrenamiento y capacidades físicas, fisiológicas y mecánicas, y 3) otras temáticas investigadas. Se confirma el hallazgo de un número alto de investigaciones vinculadas a aspectos técnicos y tácticos del fútbol sala y al análisis de las capacidades físicas. Se detectó cierta ausencia de estudios vinculados al rendimiento mental y emocional, así como estudios del rol del entrenador como responsable del equipo o trabajos sobre fútbol sala femenino, ámbitos que deben abordarse en futuras investigaciones sobre este deporte.

Palabras clave: fútbol sala, alto rendimiento, fútbol sala profesional, fútbol sala de élite.

\section{Abstract}

Futsal is a sport with a significant number of practitioners. Its small playing field and the rapidity of the players' actions encourage research on its configuration parameters to learn strategies that improve sports performance. The objective of this paper is to review the articles published between 2015 and 2020 that deal with elite futsal. A bibliographic search was carried out in the Scopus and Dialnet databases using different combinations of the terms: "futsal", "futsal", "elite", "professional" and "high performance". A total of 114 articles were found, and 50 papers were analyzed after applying the inclusion criteria. Three main categories were established: 1) investigations related to game conditioning factors, 2) investigations related to training loads and physical, physiological and mechanical capacities, and 3) other investigated topics. The finding of a high number of investigations related to technical and tactical aspects of futsal and the analysis of physical capacities is confirmed. A certain absence of studies related to mental and emotional performance was detected, as well as studies of the coach's role as head of the team or work on women's futsal, areas that should be addressed in future research.

Key words: futsal, high performance, professional futsal, elite futsal. 


\section{Introducción}

El fútbol sala es un deporte moderno que se caracteriza por ser un juego muy rápido con veloces transiciones que generan continuas ocasiones de gol (Álvarez et al., 2019), lo cual genera que sea un deporte atractivo para el espectador. Los equipos, tanto a nivel nacional como internacional, cada vez están más igualados por lo que el análisis y comprensión de lo que ocurre en la competición es interesante para poder tener una ventaja en la competición (Álvarez et al., 2019). El fútbol sala se caracteriza por ser un deporte de equipo, en forma de cooperación y oposición, donde los jugadores de los equipos compiten para marcar gol y evitar que se lo marquen para ganar el partido (da Silva Barbosa et al., 2019).

Cualquier detalle durante un partido de fútbol sala, por pequeño que parezca, puede marcar la diferencia entre dos equipos (Bortolini, \& Soares, 2018). Sin embargo, tiene un déficit de publicaciones científicas con respecto a otras modalidades de equipo, como pueden ser fútbol, baloncesto, balonmano o waterpolo, siendo necesarios más estudios que permitan comprender mejor todo lo que ocurre en la competición (Agras et al., 2016; Gómez Ruano et al., 2018; Moore et al., 2014), si bien en los últimos años han aumentado los estudios, siendo diversos los objetos de investigación. De hecho, el análisis del rendimiento técnico-táctico ha adquirido una gran importancia, sin duda el análisis del rendimiento desde un punto de vista científico puede mejorar diferentes aspectos de un equipo, por ejemplo, a partir del entrenamiento y la evaluación de las acciones a balón parado, ya que es uno de los factores más analizados por los equipos (García-Angulo \& García-Angulo, 2018) y que en la actualidad está dando sus frutos en cuanto a resultados en el alto rendimiento en fútbol sala.

A lo largo de los años se han desarrollado varios métodos para controlar los volúmenes de entrenamiento y optimizar el rendimiento del jugador. La prevención de lesiones se ha convertido en una prioridad para el cuerpo técnico. Algunos autores consideran el volumen, la intensidad y la frecuencia del entrenamiento como claves para la prevención de lesiones deportivas en los jugadores (Álvarez, Murillo, Giménez et al., 2016). En el proceso de mejorar el rendimiento los métodos de recuperación deben optimizarse. Así, se requiere una rápida recuperación para maximizar el rendimiento en el entrenamiento, la competición y aminorar la fatiga, recuperándose lo más rápido posible (Pelana et al., 2019).

En el ámbito del rendimiento mental de los jugadores existen pocos estudios científicos sobre distintas facetas de la personalidad asociadas con el comportamiento impulsivo, que pueden ayudar a los entrenado- res a optimizar su rendimiento deportivo (Castillo-Rodríguez et al., 2018). Algunos autores consideran que el fútbol sala es un deporte con creciente popularidad y nivel de rendimiento (Barbero-Álvarez et al., 2015; Lago, Jiménez et al., 2020). Sin duda, se abre una relevante prospectiva de estudio sobre los factores y cómo influyen sobre la fatiga mental en los jugadores de fútbol de alto nivel de rendimiento.

Por otro lado, no se han encontrado muchos estudios que hayan investigado las demandas fisiológicas específicas de las jugadoras de fútbol sala (Barbero-Álvarez et al., 2015) y las características de rendimiento de los jugadores (Naser \& Ali, 2016). Por ello, se precisa de más investigación en este sentido, para entender mejor los factores multifactoriales que son los causantes de las lesiones de los jugadores. En este sentido, se hace necesario el estudio del efecto que producen las cargas para intentar optimizar el rendimiento, y de este modo prevenir lesiones debidas tanto al sobreentrenamiento, como a la falta de este, y llegar a la competición con las mayores garantías y mejor estado de forma posible (Álvarez, Murillo, Giménez et al., 2016).

En una revisión bibliométrica sobre este deporte se hallaron un gran número de artículos de tipo experimental, vinculados principalmente al sexo masculino y a deportistas de nivel profesional. Respecto a las instituciones, tanto a las federaciones como por sus ligas nacionales, destacan las de países con un gran auge en lo que se refiere a fútbol sala, como Brasil y Portugal (Palazón et al., 2015). Esto también puede ser debido al crecimiento del número de los investigadores y la mejora de la calidad en las ciencias del deporte en estos dos países en las últimas décadas.

La identificación y el desarrollo de jugadores talentosos es una prioridad dentro de los clubes de fútbol sala. Esta perspectiva debe tenerse en cuenta desde una perspectiva multifactorial, pues son muchos los elementos que condicionan el desarrollo de los jugadores talentosos. Entre otras líneas de mejora e investigación que se deben estudiar en profundidad están los métodos de entrenamiento basados en juegos reducidos en fútbol sala (Hulka, \& Weisser, 2017), o conocer mejor las similitudes y diferencias entre los juegos reducidos y el juego global 5 vs 5 (Amani-Shalamzari et al., 2019), que se utilizan comúnmente para ayudar al aprendizaje y desarrollo del jugador. Es fundamental que los profesionales guíen y ayuden en todo momento, dentro y fuera de la pista, a los jóvenes jugadores que empiezan a destacar por sus habilidades en el fútbol sala.

Por todo lo anteriormente expuesto, el objetivo de este trabajo es realizar una revisión sistemática de la literatura científica publicada entre enero del año 2015 y agosto del 2020 que aborde el ámbito del fútbol 
sala de alto rendimiento, seleccionando para el análisis de contenido solamente los artículos relacionados con el alto rendimiento o los jugadores de élite, lo que facilitará tener un marco de evidencias científicas y detectar posibles carencias en el ámbito de la investigación en este deporte.

\section{Metodología}

La revisión bibliográfica es un tipo de artículo científico que recopila la información más relevante sobre un determinado ámbito o tema. En esta revisión se ha realizado una búsqueda bibliográfica en dos importantes bases de datos, una base de datos internacional como es Scopus (Elsevier), con más de 70 millones de artículos en el año 2019, y otra base de datos española, como es Dialnet, la cual recopila más de 7 millones de documentos científicos. Para la elección de los descriptores a utilizar en los motores de búsqueda se utilizaron diversas combinaciones de los términos registrados en el Tesaurus de la UNESCO [futsal, fútbol sala, alto rendimiento, élite y profesional]. Los criterios de inclusión utilizados en la revisión fueron los siguientes:

1) Artículos publicados desde el 1 de enero de 2015 hasta el 30 de agosto del 2020.

2) Que aborden cualquier tipo de investigación vinculada al fútbol sala en el contexto internacional, incorporando estudios experimentales, descriptivos, cuasi-experimentales y/o estudios de caso.

3) Artículos que fueron publicados en inglés, portugués o español.

4) Que el estudio se realizase con una muestra de jugadores de alto rendimiento, de élite o profesionales, analizando alguna variable relacionada con este ámbito.

En la figura 1 se puede observar el diagrama de flujo aplicado.
En la primera fase se realizó la búsqueda y selección de los descriptores a través del Thesaurus de la UNESCO. Posteriormente se aplicaron los descriptores con diferentes operadores boleanos (futsal o fútbol sala y alto rendimiento/élite/profesional) en las bases de datos Scopus y Dialnet. En este proceso se detectaron inicialmente 79 artículos en Scopus y 35 trabajos en Dialnet, sumando un total de 114 trabajos. Después se pasó el filtro para eliminar artículos duplicados y se obtuvieron 11 duplicidades. Por último, se aplicaron los criterios de inclusión quedando en total para el análisis de contenido final y revisión 50 trabajos. Se realizó el análisis del contenido de los artículos y clasificación por temáticas, procediéndose a estudiar detenidamente el contenido de los artículos leyendo todo el trabajo desarrollado por los investigadores y estableciendo así las diferentes categorías elaboradas para sintetizar la información en este manuscrito de revisión.

Es importante tener en consideración que, debido a la diversidad metodológica, tipos y diseños de los estudios, diferentes muestras analizadas (niveles de pericia, jugadores de clubes o de selecciones nacionales, campeonatos de diferente duración, etc.), los resultados, discusión y conclusiones deben tomarse con cautela, aunque bien es cierto que las evidencias científicas sobre fútbol sala han mejorado significativamente tanto en cantidad como en calidad en los últimos años, buena prueba de ello es la presente revisión sistemática.

\section{Resultados}

Para cumplir con el objetivo de esta revisión, que es analizar la literatura científica vinculada al fútbol sala de alto rendimiento publicada en los años 2015-2020, se han establecido tres categorías para clasificar los artículos con los que se trabajó:

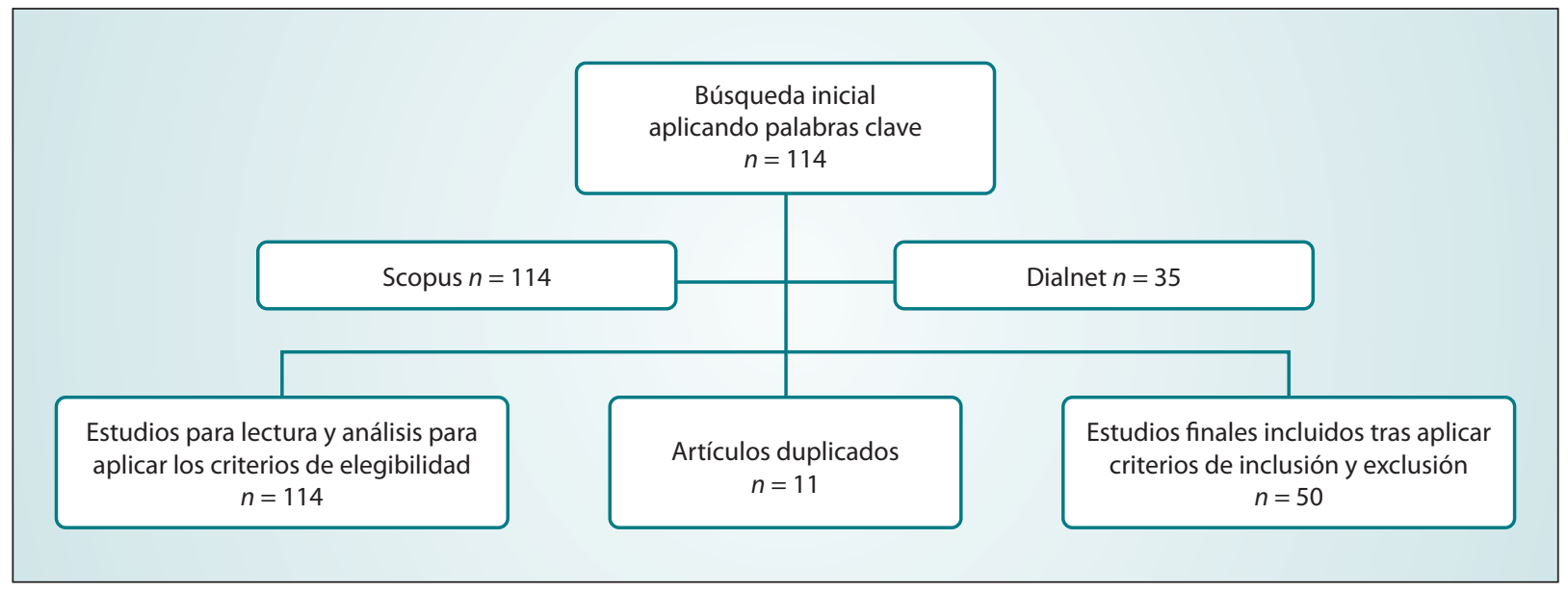

Figura 1. Diagrama de flujo del proceso de la revisión sistemática. 
Tabla 1. Síntesis de los artículos encontrados en la revisión sistemática sobre fútbol sala de alto rendimiento deportivo.

\begin{tabular}{|c|c|c|}
\hline Categorías & Número de artículos & Autores y años de publicación \\
\hline $\begin{array}{l}\text { Investigaciones } \\
\text { relacionadas con } \\
\text { condicionantes del juego }\end{array}$ & 21 & $\begin{array}{l}\text { Álvarez, Parra et al. (2018); Álvarez et al. (2018a); Álvarez et al. (2018b); Álvarez et } \\
\text { al. (2019); Álvarez, Murillo et al. (2020); Álvarez, Ramírez et al. (2020); Bortolini \& } \\
\text { Soares (2018); Campos et al. (2015); Cuasapud \& Hurtado (2018); García-Angulo \& } \\
\text { García-Angulo (2018); Gómez et al. (2018); Gómez et al. (2015); Giani et al. (2018); } \\
\text { ledynak et al. (2019); Méndez, Gómez \& Ruíz-Pérez (2019); Méndez-Domínguez, } \\
\text { Gómez-Ruano, Ruíz-Pérez \& Travassos (2019); Méndez et al. (2019b); Méndez, } \\
\text { Travassos, Santos, Ribeiro \& Gonçalves (2019); Pascual et al. (2019); Sarmento et al. } \\
\text { (2016); Vieira et al. (2016); }\end{array}$ \\
\hline $\begin{array}{l}\text { Investigaciones } \\
\text { relacionadas con cargas de } \\
\text { entrenamiento y trabajo } \\
\text { de las capacidades físicas, } \\
\text { fisiológicas y mecánicas }\end{array}$ & 17 & $\begin{array}{l}\text { Álvarez \& Murillo (2016); Álvarez, Murillo, Usán et al. (2016); Caetano et al. } \\
\text { (2015); de Freitas et al. (2019); Farhani et al. (2019); Giulano et al. (2015); Guachi } \\
\text { \& Cerón (2019); Ibáñez et al. (2020); Miloski et al. (2016); Nakamura et al. (2016a); } \\
\text { Nakamura et al. (2016b); Naser \& Ali (2016); Nunes et al. (2016); Paz et al. (2017); } \\
\text { Sánchez-Sánchez et al. (2018); Wilke et al. (2016); Wilke et al. (2020) }\end{array}$ \\
\hline $\begin{array}{l}\text { Otras temáticas } \\
\text { investigadas }\end{array}$ & 12 & $\begin{array}{l}\text { Almeida et al. (2019); Amorim et al. (2020); Carolina-Paludo et al. (2020); Castillo- } \\
\text { Rodríguez et al. (2018); de Oliveira et al. (2020); Lago, Rey et al. (2020); Nascimento } \\
\text { et al. (2017); Nascimento et al. (2018); Nascimento et al. (2019); Sarmento et al. } \\
\text { (2015); Sezen-Balçikanli \& Sezen (2017) Pascual Verdú et al. (2016). }\end{array}$ \\
\hline
\end{tabular}

- Investigaciones relacionadas con condicionantes del juego en el fútbol sala profesional.

- Investigaciones vinculadas a cargas de entrenamiento y trabajo de las capacidades físicas, fisiológicas y mecánicas en el fútbol sala profesional.

-Investigaciones vinculadas a otras temáticas en el fútbol sala profesional

En tabla 1 se recopila la información del número de artículos encontrados en cada categoría, sus autores de referencia y año de publicación.

La revisión de la producción científica vinculada al fútbol sala profesional en los años 2015-2020 confirma que principalmente la atención de los investigadores se centró en el análisis de los condicionantes de juego, con un total de 21 trabajos. El estudio de las cargas de entrenamiento y trabajo de las capacidades físicas también tuvo un destacado protagonismo, con 17 artículos de referencia. Dentro de los trabajos analizados en la categoría otras temáticas se registró un número bajo de estudios vinculados a la esfera emocional o psíquica de los jugadores, incorporándose en este grupo también estudios vinculados a la gestión del equipo humano por parte del entrenador y un trabajo sobre fútbol sala femenino.

\section{Discusión}

Investigaciones relacionadas con los condicionantes del juego

En el fútbol sala el gol es el indicador por excelencia del rendimiento en los distintos campeonatos. Cuasapud y Hurtado (2018) mostraron una asociación sig- nificativa entre anotar el primer gol y ganar el juego. Así pues, la lógica interna del futbol sala está formada por ataque y defensa que implican continuas transiciones ofensivas y defensivas y ocasiones de gol. Esta movilidad en ataque tiene como finalidad provocar y aprovechar un desequilibrio en el esquema táctico defensivo contrario, creando así un efecto sorpresa, de incertidumbre, que genere una opción clara de finalización y así poder anotar gol (Álvarez, Ramírez et al., 2020). Los resultados de otros autores, como Méndez, Travassos et al. (2019), permitieron entender que los equipos portugueses y rusos utilizaron más los ataques posicionales y menos los contraataques y jugadas a balón parado que los españoles, que presentan un perfil ofensivo más equilibrado. Álvarez, Murillo et al. (2020) indicaron que los momentos con más goles del partido fueron en los últimos 10 minutos de la segunda parte y los últimos cinco minutos de la primera parte y que estos patrones se han dado en las tres mejores ligas europeas de fútbol sala. Por otra parte, Gómez et al. (2015) identificaron la importancia de la efectividad de las posesiones de balón en la élite aumentado esta durante jugadas a balón parado y utilizando más de cuatro pases.

Álvarez et al. (2019) estudiaron los goles en las diferentes ligas, siendo la liga rusa la más goleadora, con una media por partido de 8,16 goles, seguida por la española, con 7,37 goles, y la italiana, con 6,67 goles. Los equipos locales anotan el 54,2\% de los goles, por el $45,8 \%$ como visitante. En cambio, Giani et al. (2018) analizaron 1875 goles de los 259 partidos de la Liga Nacional de fútbol sala española (LNFS) durante la temporada 2015/2016 y constataron que el ataque posicional fue la acción técnico-táctica más recurrente en los goles ligueros, con el $34 \%$ de los goles, seguido del 
contraataque, con el $26 \%$, y juego a balón parado, con el 22\%. El uso de la línea de ataque del portero resultó ser eficiente, ya que el $8 \%$ de los goles marcados en la competición fue de esta forma, mientras que el $6 \%$ fue con la línea de marcaje del portero, donde este jugador está más retrasado (Giani et al., 2018). Para entender mejor estos conceptos, en el juego de la línea de ataque del portero, donde este jugador adelanta su posición, se lleva a cabo pases más cortos con una mayor tasa de eficacia, consiguiendo la "aceleración de los pases" y mayor velocidad de la fase ofensiva, y obligando de este modo a un retraso en el marcaje del equipo rival y, por tanto, que los jugadores defensivos tengan dificultades para encontrarse en situación de recuperación del balón.

En este sentido, Sarmento et al. (2016) apuntan que se marcaron más goles en la LNFS en acciones de ataque posicional (42\%) y desde jugadas a balón parado (27\%) en comparación con otras actividades. Las áreas zonales ofensivas centrales fueron las más comunes para los tiros a puerta, con el $73 \%$ de todos los goles marcados desde estas áreas del campo en comparación con las zonas defensivas y amplias, siendo el pie la parte principal del cuerpo involucrada en la puntuación.

De todos es conocida la relevancia del juego a balón parado para el rendimiento en el fútbol sala. Por ejemplificar esta línea de estudio en un análisis de los córners $(n=364)$ que se producen en el Campeonato de la UEFA 2016: el 55,55\% de los goles de saques de esquina se consiguen en los últimos diez minutos de la primera parte. Ello debe de tener influencia en la forma de defender los córners, en este campeonato la mayoría de los saques de esquina fueron defendidos en defensa zonal (García-Angulo \& García-Angulo, 2018). En otro estudio, en este caso tomando como muestra los ocho equipos finalistas de la edición 2016 de la Liga Nacional brasileña masculina, Bortolini y Soares (2018) confirmaron que el $26 \%$ de los goles fueron jugadas con último pase al segundo poste, demostrando una alta influencia en el resultado final de los partidos, la acción ofensiva que resultó en el mayor número de jugadas completadas con goles al segundo poste fue a través del contraataque, con el 42,1\% de los goles. Es importante recalcar la dificultad de comparar y discutir las acciones de juego de unos contextos a otros, o de los estudios realizados en campeonatos con características diferentes, pues ello modifica la forma y el estilo de juego de los equipos y, por consiguiente, la forma de conseguir los goles; no es lo mismo analizar una competición de selecciones nacionales en el ámbito internacional (García-Angulo \& García-Angulo, 2018) que una liga regular de un determinado país (Bortloni \& Soares, 2018).
Álvarez, Parra et al. (2018) analizaron las acciones ofensivas que terminaron en gol a lo largo de las temporadas 2012-2013 y 2013-2014 en la LNFS, determinando las relaciones entre las variables anteriores que están involucradas en estas acciones. Los resultados de estos autores señalan que la mayoría de los goles en fútbol sala se producen en jugadas rápidas de 1-2 pases con 1-2 jugadores participantes y que con defensas organizadas se requiere un mayor número de pases y la participación de tres jugadores para encontrar líneas de pase antes de disparar con opciones de hacer gol.

Pascual et al. (2019) estudiaron un total de 840 goles pertenecientes a la temporada 2016/2017 y no detectaron cambios significativos en las variables zona del campo, tiempo de juego y superficie de contacto con respecto a conocer en profundidad las variables más utilizadas por los jugadores para que se produzca un gol. Si se saben cuáles son las variables determinantes en las jugadas que finalizan en gol estas pueden ser usadas para desarrollar metodologías de entrenamiento más eficaces en función del objetivo pretendido. Los resultados de otra investigación sobre el análisis de cómo se consiguen los goles en las ligas europeas (española, rusa e italiana, durante la temporada 2014-2015, con un total de 4716 goles) establecen que el jugador en la posición de pívot es el que obtiene un 40,37\% de los goles, seguido por los jugadores en posición de ala, con un 37,24\% y los jugadores en posición de cierre, con un 19,64\% (Álvarez, Ramírez et al., 2020). Sin embargo, en el estudio de Álvarez et al. (2018a), donde se analizan un total de 1755 goles de la Liga Nacional de Fútbol Sala española (2014-15), los jugadores en posición de ala consiguen el $54,1 \%$, los pívots el $29,5 \%$, los cierres el $15,8 \%$ y los porteros el $0,5 \%$ de los goles. Como se puede observar, hay diferencias según el contexto (liga analizada) y tipo de juego, sobre todo en las posiciones de ala y pívot, aunque se confirma que son las posiciones de juego donde más goles se anotan.

Otra línea de estudio ha sido el análisis de juego cuando se utiliza el rol de portero jugador. En este sentido, Méndez et al. (2019a) identificaron la importancia y el significado de los goles utilizando al portero jugador en la élite del fútbol sala. Sus resultados confirmaron que había una mayor probabilidad de marcar goles cuando los equipos estaban ganando, en partidos equilibrados y en los últimos ocho minutos de partido, por tanto, son situaciones de partido que deben ser entrenadas para mejorar el rendimiento de los equipos. Por el contrario, observaron una mayor probabilidad de recibir goles utilizando al portero jugador cuando los equipos iban perdiendo en partidos desequilibrados y en los últimos ocho minutos, lo que 
responde a la lógica, porque el equipo que va perdiendo asume riesgos defensivos para tratar de remontar el partido. De igual manera, se debe entrenar el aspecto defensivo de las acciones de juego cuando se participa con el rol de portero jugador.

El portero jugador viene siendo utilizado por la mayoría de los entrenadores de fútbol sala en situaciones de emergencia y relacionadas con un marcador adverso en los momentos finales del partido (Méndez, Gómez \& Ruíz-Pérez, 2019). Los resultados de estos autores enfatizaron la relación de simultaneidad que parece existir entre la utilización del portero jugador y un marcador desfavorable cuando queda poco tiempo para finalizar, lo que es sinónimo de tener que afrontar una situación crítica a través de un procedimiento táctico de riesgo. Méndez et al. (2019b) indagaron en la importancia de la posibilidad de que el portero dispare a portería como jugador con una muestra de 11.446 acciones correspondientes a 1325 partidos de la LNFS en España (temporadas 2010-2015). Sus resultados mostraron relaciones de éxito cuando los equipos jugaron en casa, contra un oponente clasificado en una posición similar, durante partidos equilibrados, y tuvieron hasta dos faltas. Cuando los equipos empataban o perdían, las acciones del portero jugador no tenían éxito. Además, identificaron una mayor probabilidad de disparar en partidos equilibrados durante los playoffs, y con no más de dos faltas. Estos análisis científicos son importantes para que los entrenadores preparen los partidos y apliquen o no este tipo de estrategias ofensivas.

La ventaja de jugar en casa se ha observado en diferentes deportes individuales y de equipo. Los resultados sugieren la existencia de un efecto de ventaja del local en fútbol sala profesional $(63,8 \%)$, independientemente de la calidad del oponente. Las tendencias identificadas confirman la influencia de la ventaja de jugar en casa en fútbol sala y la relación negativa con equipos más fuertes (Campos et al., 2015). Siguiendo líneas de estudio similares, Gómez et al. (2018), a partir de un análisis de 125 situaciones de juego de ataque en superioridad numérica (por exclusión de oponentes del equipo contrario) de la temporada regular de la LNFS y con el propósito de estudiar el efecto de las exclusiones de jugadores en el resultado de los ataque en los partidos de fútbol sala de élite analizando el efecto de las variables relacionadas con el contexto (calidad del adversario, localización del partido, períodos de juego, faltas del equipo contarios, estado del partido, duración del ataque, y tipo de partido), mostraron que la mayor efectividad de ataque se logró cuando los equipos juegan en casa, realizan el ataque durante los minutos 33-36 y el equipo contrario tiene tres faltas, e identificaron cuatro tipos de ataques cuando los equipos jugaban con superioridad numérica. Sin embargo, Álvarez et al. (2018b) no obtuvieron diferencias significativas al comparar jugar como local o visitante. Jugar como local aumenta un $18,15 \%$ las posibilidades de ganar el partido. De las veces que se llega al descanso con un marcador a favor se obtiene un $83,48 \%$ de posibilidades de ganar el partido como local y un $74,66 \%$ como visitante y si el marcador parcial no es de derrota las probabilidades pasan a un $89,90 \%$ como local y un $82,66 \%$ como visitante (Álvarez et al., 2018b).

Otra línea de investigación relevante ha sido el estudio de la organización en la pista, el rendimiento físico y técnico de los jugadores profesionales de fútbol sala. Para ejemplificar esta línea de estudio se aborda el artículo de Vieira et al. (2016), donde se analizaron a diez jugadores profesionales de fútbol sala de Brasil. Los partidos fueron monitorizados con cámaras de vídeo $(30 \mathrm{~Hz})$ y las trayectorias de los jugadores se obtuvieron con un seguimiento automático. El objetivo principal de este estudio fue analizar las posibles diferencias entre un partido amistoso de pretemporada y un partido oficial de temporada en cuanto al desempeño físico, técnico y organizativo. Así pues, los valores obtenidos para la distancia recorrida por minuto, porcentaje de distancia recorrida a intensidad moderada, área de cobertura del equipo, spread, pases, posesiones, toques de balón y pases exitosos por minuto fueron mayores para el partido oficial que para el amistoso de pretemporada. Por el contrario, el porcentaje de distancia recorrida, de pie y caminando fue mayor para el partido amistoso que para el oficial. Por tanto, en el futuro se debería verificar si los torneos de pretemporada reproducen demandas similares a las de un partido oficial de la temporada regular, o si los entrenamientos que se realizan durante la semana responden de manera similar o diferente a las demandas físicas y técnico-tácticas que luego se precisan en los partidos de competición.

En esta misma línea de investigación, Iedynak et al. (2019) analizaron las características cuantitativas de los tipos de movimientos que se utilizan por los jugadores profesionales de fútbol sala, pero con diferentes roles de posición durante las actividades competitivas. Este objetivo fue obtenido a través del seguimiento de 36 jugadores profesionales de la primera división de Ucrania durante 16 partidos, donde se usaron los siguientes métodos: grabación en vídeo, cronometría y midiendo la longitud del paso. De esta manera, los resultados sobre la distancia recorrida según las posiciones de los jugadores de fútbol sala fueron que la distancia más larga fue realizada por los jugadores con el rol de pívot, mientras que en la fase defensiva 
cubrieron una distancia mucho más corta, pero siendo esta distancia mayor que la de los porteros. Durante el juego, los pívots trotan y esprintan con más frecuencia que en la fase defensiva, y estos caminan y corren con más frecuencia que los delanteros. Los resultados de este estudio determinan tanto los puntos fuertes como las debilidades en la actividad locomotora de cada jugador en los equipos profesionales, por lo que se analizan las posibilidades de mejora, y de esta forma, los entrenadores pueden beneficiarse de estos resultados para entender cómo se organizan los jugadores con diferentes roles durante los partidos oficiales y usar esta información para mejorar los programas de entrenamiento.

Investigaciones relacionadas con cargas de entrenamiento y trabajo de las capacidades físicas, fisiológicas y mecánicas

La enseñanza de la técnica deportiva en el proceso de dirección del entrenamiento deportivo es esencial para el logro del alto rendimiento (Guachi \& Cerón, 2019). Los resultados de otra investigación realizada por Álvarez y Murillo (2016) con 12 jugadores profesionales de fútbol sala español durante toda la temporada (40 semanas) muestran que la relación de la percepción de la intensidad del entrenamiento de los jugadores con la carga planificada por el cuerpo técnico (denominado como doble escala) permite comprobar y establecer la desviación entre la percepción del jugador y del cuerpo técnico, así como establecer cómo va asimilando las cargas el jugador posibilitando reajustar la misma según la información recibida. Con la misma muestra (12 jugadores españoles de alto nivel) y duración del estudio (una temporada: 40 semanas), Álvarez, Murillo, Usán et al. (2016) analizaron las cargas de entrenamiento en fútbol sala utilizando el método de percepción subjetiva (Escala de percepción de Borg), encontrando correlaciones significativas entre la percepción de fatiga previa y posterior, así como entre la percepción de intensidad y de fatiga posterior. Este tipo de estudios es importante, pues el método es fácilmente replicable en cualquier equipo de fútbol sala, dado que no es necesario tener aparataje que suponga un coste económico.

Sobre las cargas de entrenamiento, los hallazgos de Nakamura et al. (2016a), a partir de un estudio que compara las diferencia del rendimiento físico (medido a partir de varios tests: YO-yo IR1, sprint de 20 metros, saltos: SJ, CMJ y JS, etc.) entre los jugadores brasileños de alto nivel de categoría sub-20 (15 jugadores) y los de categoría absoluta (18 jugadores senior), señalan que la práctica continuada de fútbol sala puede conducir a una mejora en la aptitud aeróbica y la regulación autonómica cardíaca, al tiempo que afecta la potencia muscular y el rendimiento de velocidad de los jugadores.

Este tipo de estudios corrobora la importancia del control de las cargas de entrenamiento como prevención de lesiones y disminución de la incidencia lesional, siendo actualmente uno de los objetivos fundamentales del cuerpo técnico, ya que un jugador lesionado no ayuda al rendimiento del equipo ni en los entrenamientos ni en la competición. Por este y otros motivos, Miloski et al. (2016) describieron la distribución de la carga de entrenamiento de un equipo profesional de 12 jugadores de fútbol sala masculino con el objetivo de verificar sus efectos posteriores en el rendimiento físico, el daño muscular y el estado hormonal. El programa de carga de entrenamiento, compuesto por 22 semanas, fue más alto durante los períodos con baja incidencia de partidos, enfatizando el trabajo de la resistencia y fuerza en los entrenamientos durante la pretemporada y el entrenamiento de la velocidad y potencia durante la temporada. Nakamura et al. (2016b) evaluaron las características de velocidad-potencia en 10 jugadores de fútbol sala brasileños sub-20 antes y después de una pretemporada de 9 semanas y exploraron las posibles relaciones con cargas de entrenamiento internas. Según estos autores el entrenamiento de pretemporada conduce a un rendimiento deficiente en el salto vertical sin carga y en la prueba de velocidad, concluyendo que los jugadores de fútbol sala más rápidos perciben mayores cargas de entrenamiento y presentan mayores disminuciones en la velocidad de esprint durante la pretemporada. En cambio, Wilke et al. (2020), a partir de un estudio sobre la influencia de las clasificaciones de perfil de recuperación más rápidas y lentas, sueño autoinformado, carga de entrenamiento aguda y fase del microciclo en la recuperación percibida en 19 jugadores de élite en fútbol sala masculino, confirman que ni la clasificación de recuperación ni la carga de entrenamiento previa influyeron en la recuperación percibida durante la pretemporada; sin embargo, la recuperación más alta se produjo con una mejor calidad del sueño.

Siguiendo con esta línea de estudio, Ibáñez et al. (2020) estudiaron las diferencias existentes de cómo afecta la intensidad del entrenamiento en 9 jugadores profesionales de fútbol sala masculina pertenecientes a un equipo de primera división de la LNFS, atendiendo a la posición ocupada en el terreno de juego, y comparan los parámetros de frecuencia cardiaca entre entrenamientos y competición. Los resultados obtenidos por estos no mostraron diferencias significativas respecto a la carga cuantificada en las tres posiciones, 
además la carga interna se manifestó de forma más exigente en jugadores que ocupaban la posición de ala y pívot. Continuando con las demandas metabólicas y las cargas de entrenamiento interno en sesiones de entrenamiento, Wilke et al. (2016), a partir de un estudio de 12 jugadores de alto nivel en fútbol sala donde se les midió con tests fisiológicos (frecuencia cardíaca máxima o HRmax, umbral de ventilación o VT, y su punto de compensación respiratoria o RCP), señalaron que los jugadores con los roles de alas, cierres y pívots se ejercitaron a una intensidad media similar, pero con distribuciones de intensidad diferentes.

En otra investigación realizada por Giulano et al. (2015) en la que se analizaron 97 jugadores de fútbol sala profesional durante 5 partidos oficiales usando el sistema de seguimiento automático de DVideo se demostró un aumento en la duración del esprint desde la primera a la segunda mitad; sin embargo, no se encontraron diferencias para otras variables como la distancia recorrida, la velocidad máxima, la velocidad inicial, el tiempo de recuperación entre esprint y esprint realizado por minuto o entre posiciones de juego. Además, al considerar secuencias de esprints repetidos, los resultados mostraron que los esprints repetidos que comprenden dos esprints intercalados con un máximo de 15 segundos de recuperación fueron significativamente más frecuentes que otros esprints repetidos. Los hallazgos de este estudio sobre las características de velocidad de los jugadores de fútbol sala pueden ayudar a los entrenadores a planificar el entrenamiento físico y las evaluaciones de acuerdo con los requisitos del deporte. Siguiendo estas líneas, los resultados del estudio realizado por Caetano et al. (2015) mostraron que los esprints repetidos que comprenden dos esprints intercalados con un máximo de 15 segundos de recuperación fueron significativamente más frecuentes que otros esprints repetidos.

Para Sánchez-Sánchez et al. (2018), a partir de un estudio sobre el efecto de una prueba de habilidad de sprint repetida en las propiedades de contracción muscular con 20 jugadores de fútbol de élite, existió un incremento significativo en los tiempos de $30 \mathrm{~m}$ desde el tercer esprint en adelante, siendo el porcentaje de disminución en la capacidad de esprint con respecto al primer esprint significativamente mayor en el último. Por otro lado, Nunes et al. (2016) mostraron que la extensión concéntrica isocinética máxima de la rodilla y el torque de flexión a todas las velocidades se correlacionaron moderadamente con el rendimiento de esprint de 5 y $30 \mathrm{~m}$ y el tiempo total de esprint. Los resultados de este estudio indican que las capacidades de producción de fuerza muscular, pero no la aptitud aeróbica, están relacionadas, al menos en parte, con la capacidad de esprintar. Otros autores, como Farhani et al. (2019) o Naser y Ali (2016), compararon las diferentes características en jugadores de fútbol sala de élite y sub-élite, los resultados mostraron que la resistencia intermitente y el tiempo de rendimiento fueron superiores en los jugadores de élite en comparación con los que no lo son; además, la potencia anaeróbica fue mayor en los jugadores de élite.

\section{Otras temáticas investigadas}

En el escenario deportivo, el autoconcepto ha sido señalado como un atributo psicológico fundamental para el desempeño de entrenadores y jugadores, incidiendo sobre el control de las emociones y las expectativas de éxito o fracaso. El apoyo social se correlacionó positivamente con la receptividad social y el estilo autocrático negativamente con la actitud social (Amorim et al., 2020). En el contexto de alto desempeño en el fútbol sala la satisfacción de los jugadores con sus necesidades psicológicas básicas está asociada con la participación en los objetivos del equipo y la probabilidad de hacer amigos dentro del grupo (Nascimento et al., 2019).

El objetivo de otro trabajo realizado por CarolinaPaludo et al. (2020), donde se analizó a 14 jugadores de fútbol sala masculino de categoría sub-20 en Brasil, fue investigar el efecto del lugar de juego sobre el cortisol y el estado de ansiedad precompetitivo en los jugadores profesionales de fútbol sala. Los datos de este estudio sugieren que el lugar de juego afecta la respuesta hormonal. Por su lado, los resultados de otros autores, tales como Castillo-Rodríguez et al. (2018), en otros contextos, dado que se analizó a 111 jugadores de fútbol sala masculino de élite de la LNFS en España en la temporada 2014/2015, mostraron una menor puntuación en los rasgos de impulsividad, específicamente en la dimensión de búsqueda de sensaciones en aquellos jugadores con rol defensivo, y particularmente en los porteros, en comparación con los pívots y ala-pívots.

Nascimento et al. (2017) analizaron el papel mediador de la satisfacción de las necesidades básicas sobre el efecto de los rasgos de perfeccionismo en la cohesión social y de tareas entre 301 jugadores de élite de fútbol sala que participaron en el campeonato brasileño. La satisfacción de las necesidades básicas fomenta el impacto del perfeccionismo adaptativo en la cohesión del equipo, al tiempo que disminuye los efectos del perfeccionismo desadaptativo. Sezen-Balçikanli y Sezen (2017), en un estudio que analizó un total de 76 jugadores profesionales de fútbol sala masculino que participaron en la Liga de Fútbol Sala en Efes Pilsen (región de Ankara), demostraron que existió una re- 
lación entre la empatía y las tarjetas amarillas, rojas y las faltas. Se considera que las prácticas de empatía a desarrollar por los deportistas fomentarán la toma de perspectiva y la comunicación interpersonal entre ellos, así como el punto de vista moral y las actitudes en el deporte y reducirán las conductas agresivas y acciones egoístas.

Los entrenadores analizan y evalúan tanto a su propio equipo como al equipo al que se enfrentan, centrándose en las fortalezas, debilidades, oportunidades y siguen una lógica específica de priorizar esto en las reuniones de equipo a lo largo de la semana. Las intervenciones aplicadas vienen principalmente en forma de ejercicios de formación específicos y análisis de vídeo. Durante el juego, los entrenadores generalmente brindan retroalimentación continuamente además de comunicarse mediante gestos. Los tiempos muertos son un aspecto clave de la competición en el fútbol sala, estos se utilizan inteligentemente por los cuerpos técnicos para corregir factores estratégicos y emocionales al mismo tiempo que factores relacionados con la fatiga (Sarmento et al., 2015). Siguiendo esta línea, el propósito de otro estudio fue analizar el proceso de toma de decisiones desde la preparación hasta los momentos competitivos (Almeida et al., 2019). Los resultados de estos autores revelaron que los entrenadores comparten ideas similares al preparar sus equipos y enfocan primero su atención en la identidad de su propio equipo y, en segundo lugar, en el comportamiento y el marco del equipo contrario. También fue posible identificar señales claras de comportamiento del coaching que se pueden estructurar como claves y líneas guía para su proceso de toma de decisiones, acciones antes y durante los momentos competitivos.

Continuando la investigación sobre los entrenadores, Nascimento et al. (2018) estudiaron el efecto del estilo de liderazgo del entrenador percibido por los 120 jugadores de fútbol sala de los ocho mejores equipos que participaron en la Liga Profesional del estado de Paraná (Brasil) en 2013, donde midieron la cohesión del equipo entre los jugadores de futbol sala de élite, existiendo relaciones significativas entre el estilo de liderazgo, la cohesión social y la cohesión para tarea. Además, el estilo de liderazgo del entrenador percibido por los atletas caracterizados como democráticos y basados en el apoyo social, el refuerzo y la instrucción de entrenamiento influyó positivamente en la cohesión de la tarea y la cohesión social moderada (Nascimento et al., 2018).

Una línea de investigación que ha sido estudiada en el fútbol sala ha sido el efecto de la edad relativa en los jugadores profesionales. En este sentido, en el estudio de Lago, Rey et al. (2020) se observó una reversión re- lativa del efecto de la edad en los jugadores profesionales de fútbol sala de las nueve temporadas analizadas en la LNFS. Se detectó un efecto relativo de reversión de edad en equipos de nivel medio y alto. También se encontró una inversión relativa del efecto de la edad para las posiciones de portero y pívot. Los resultados sugieren que los jugadores que inicialmente experimentaron desventajas de madurez en los deportes más relevantes (ej. fútbol o baloncesto) pueden tener una segunda oportunidad de emerger como deportistas de alto nivel en deportes profesionales emergentes, minoritarios o menos consolidados.

Por otra parte, el estudio de Oliveira et al. (2020), efectuado a partir del análisis de las trayectorias de 258 jugadores del estado de Paraná (Brasil) (excluyendo a los porteros) compuesto por 79 jugadores de la categoría sub-15, 86 de la sub-18 y 93 de la categoría profesional, confirma que las categorías más jóvenes presentaron una mayor frecuencia de oscilación de propagación que la categoría profesional, lo que refleja la dinámica del juego entre las secuencias de ataque y defensa. Los resultados proporcionan información sobre las características del deporte y presentan una base para las intervenciones de entrenamiento adecuadas para los jugadores en cada categoría, planificando la transición futura a la siguiente categoría.

\section{Conclusiones}

Una vez analizados los 50 artículos científicos seleccionados en esta revisión sistemática sobre los estudios en el ámbito del fútbol sala de alto rendimiento entre los años 2015 y 2020, se puede afirmar que la investigación en alto rendimiento en fútbol sala está más desarrollada y es más profunda que la de años atrás, tanto en cantidad (sobre todo con muestras de jugadores en países como Brasil, España y Portugal), como en calidad (metodologías utilizadas, así como resultados y conclusiones). En cuanto a los temas que han mostrado mayor foco de interés de los investigadores, han sido los estudios sobre los condicionantes del juego, encontrándose un número importante de trabajos científicos vinculados al entrenamiento de la técnica y la táctica, a los sistemas ofensivos y defensivos, el estudio de las posibilidades de gol y la victoria en los equipos.

También se detectó un volumen considerable de trabajos vinculados a la condición y rendimiento físico de los jugadores, concretamente al estudio de las capacidades físicas demandadas por la estructura funcional y formal de este deporte. Por el contrario, se ha hecho evidente la carencia de estudios relacionados con el 
ámbito psico-social vinculado a la rama de psicología del deporte, habiendo pocas investigaciones que abordaran el rendimiento emocional y psíquico o la fatiga mental de los jugadores, así como el estudio del liderazgo o gestión de los cuerpos técnicos de los equipos. También se ha observado la escasez de trabajos que traten de la formación y detección del talento en el alto rendimiento en el fútbol sala o investigaciones que estudien el fútbol sala profesional femenino.

\section{Aplicaciones prácticas y futuras investigaciones}

Estos resultados son de gran interés para todos los profesionales del fútbol sala, así como para el uso de buenas prácticas vinculadas a este deporte: entrenadores, preparadores físicos, fisioterapeutas, analistas de juego, psicólogos, médicos deportivos e investigadores en ciencias del deporte, pues conocerán cuáles son los puntos fuertes en el presente $y$, a su vez, podrán tener más claras las prospectivas para abordar nuevos estudios en el futuro con el objetivo de conseguir mayor investigación en todas las esferas inherentes al máximo rendimiento en el fútbol sala.

De cara a futuros trabajos que se desarrollen en el fútbol sala es recomendable aportar estudios vinculados al ámbito de la psicología, sociología y pedagogía deportiva para conocer más las exigencias emocionales, psíquicas y de gestión de relaciones sociales que se producen en el fútbol sala, pues pueden contribuir a la mejora de este deporte y a un mayor rendimiento deportivo. Además, sería pertinente una mayor investigación en el fútbol sala femenino, que, como se ha podido observar, no se ha abordado profundamente en los últimos años.

\section{B I B L I O G R A Fí A}

Agras, H., Ferragut, C., \& Abraldes, J. A. (2016). Match analysis in futsal: a systematic review. International Journal of Performance Analysis in Sport, 16(2), 652-686. https://doi.org/10.1080/24748668.2016.11868915

Almeida, J., Sarmento, H., Kelly, S., \& Travassos, B. (2019). Coach decision-making in futsal: from preparation to competition. International Journal of Performance Analysis in Sport, 19(5), 711-723. https://doi.or g/10.1080/24748668.2019.1648717

Álvarez Medina, J., \& Murillo Lorente, V. (2016). Comparación entre las cargas planificadas y ejecutadas en el entrenamiento de fútbol sala: la doble escala. Retos: Nuevas Tendencias en Educación Física, Deporte y Recreación, 29, 48-52.

Álvarez Medina, J., Murillo Lorente, V., Amatria Jiménez, M., \& Ramírez San José, J. (2020). Momentos críticos del partido en las mejores ligas europeas de fútbol sala. Retos: Nuevas Tendencias en Educación Física, Deporte y Recreación, 38, 77-82.

Álvarez Medina, J., Murillo Lorente, V., Giménez Salillas, L., \& Manonelles Marqueta, P. (2016). Modificación del volumen-intensidad como medida preventiva de lesiones en fútbol sala. Revista Internacional de Medicina y Ciencias de la Actividad Física y del Deporte/International Journal of Medicine and Science of Physical Activity and Sport, 16(61), 85-97. https://doi.org/10.15366/rimcafd2016.61.007

Álvarez Medina, J., Murillo Lorente, V., Usán Supervía, P., Manonelles Marqueta, P., \& Ros Mar, R. (2016). Percepción subjetiva como método de control de la fatiga y la intensidad en fútbol sala. Retos: Nuevas Tendencias en Educación Física, Deporte y Recreación, 30, 9-14.

Álvarez Medina, J., Parra Artal, A., García Felipe, A., \& Murillo Lorente, V. (2018). Análisis observacional de los goles de dos temporadas de la LNFS.

Álvarez Medina, J., Ramírez San José, J., \& Murillo Lorente, V. (2018a). Influencia de la posición inicial en la consecución de los goles de la Liga Nacional de fútbol sala. Revista Internacional de Deportes Colectivos, 35, 5-13.

Álvarez Medina, J., Ramírez San José, J., \& Murillo Lorente, V. (2018b). Importancia de llegar al descanso con el marcador a favor en fútbol sala half time score influence in fútbol sala final score. Revista Internacional de Deportes Colectivos, 34, 5-12.

Álvarez Medina, J., Ramírez San José, J., \& Murillo Lorente, V. (2019). El gol como unidad de medida de rendimiento en fútbol sala. Retos: Nuevas Tendencias en Educación Física, Deporte y Recreación, 36, 251-258.

Álvarez Medina, J., Ramírez San José, J., \& Murillo Lorente, V. (2020). Efectividad de los jugadores de fútbol sala según sus posiciones. $R e$ tos: Nuevas Tendencias en Educación Física, Deporte y Recreación, 37, $147-151$
Amani-Shalamzari, S., Khoshghadam, E., Donyaei, A., Parnow, A., Bayati, M., \& Clemente, F. M. (2019). Generic vs. small-sided game training in futsal: Effects on aerobic capacity, anaerobic power and agility. Physiology \& behavior, 204, 347-354. https://doi.org/10.1016/j. physbeh.2019.03.017

Amorim, A. C., do Nascimento Junior, J. R. A., Contreira, A. R., Granja, C. T. L., \& Vieira, J. L. L. (2020). O autoconceito é um atributo interveniente no estilo de liderança de treinadores de fútbol sala de alto rendimento? Revista Iberoamericana de Psicología del Ejercicio y el Deporte, 15(1), 7-11.

Barbero-Alvarez, J. C., Subiela, J. V., Granda-Vera, J., Castagna, C., Gómez, M., \& Del Coso, J. (2015). Aerobic fitness and performance in elite female fútbol sala players. Biology of Sport, 32(4), 339. https:// doi.org/10.5604/20831862.1189200

Bortolini, C. M., \& Soares, B. H. (2018). Análise da origem e incidência dos gols de $2^{\text {a }}$ trave no Fútbol sala. RBFF-Revista Brasileira de Fútbol sala e Futebol, 10(41), 751-758.

Caetano, F. G., de Oliveira, M. J., Marche, A. L., Nakamura, F. Y., Cunha, S. A., \& Moura, F. A. (2015). Characterization of the sprint and repeated-sprint sequences performed by professional futsal players, according to playing position, during official matches. Journal of Applied Biomechanics, 31(6), 423-429. https://doi.org/10.1123/ jab.2014-0159

Campos, F. A., Pellegrinotti, Í. L., Pasquarelli, N. B., Rabelo, N. F., SantaCruz, R. A., \& Gómez, M. Á. (2015). Effects of game-location and quality of opposition in futsal league. International Journal of Performance Analysis in Sport, 15(2), 598-607. https://doi.org/10.1080/247 48668.2015.11868817

Carolina-Paludo, A., Nunes-Rabelo, F., Maciel-Batista, M., Rúbila-Maciel, I. Peikriszwili-Tartaruga, M., \& Simões, A. C. (2020). Game location effect on pre-competition cortisol concentration and anxiety state: A case study in a futsal team. Revista de Psicología del Deporte, 29(1), 105-112.

Castillo-Rodríguez, A., Madinabeitia, I., Castillo-Díaz, A., Cárdenas, D., \& Alarcón, F. (2018). The impulsivity determines the role performed by futsal players. Revista de Psicologia del Deporte, 27(2), 181-188.

Cuasapud, D. A., \& Hurtado, H. (2018). Impacto del primer gol: Copa Mundial Fútbol sala FIFA Colombia 2016. RBFF-Revista Brasileira de Fútbol sala e Futebol, 10(40), 544-549.

da Silva Barbosa, A. K., da Silva Caldas, E., da Silva, D. M. B., Araújo, M L., \& Navarro, A. C. (2019). Avaliação de hábitos alimentares de uma equipe de fútbol sala feminino. RBNE-Revista Brasileira de Nutrição Esportiva, 13(79), 283-288. 
de Freitas, V. H., Rinaldo, M., Turquino, G. G., Miloski, B., \& Ramos, S. D. P. (2019). Training aimed at the development of power and physical performance of futsal players. Revista Brasileira de Cineantropometria \& Desempenho Humano, 21, e60119. https://doi.org/10.1590/19800037.2019v21e60119

de Oliveira Bueno, M.J., Caetano, F.G., de Souza, N.M., Cunha, S.A., \& Moura, F.A. (2020). Variability in tactical behavior of futsal teams from different categories. PloS one, 15(3), e0230513. https://doi. org/10.1371/journal.pone.0230513

Farhani, F., Rajabi, H., Negaresh, R., Ali, A., Shalamzari, S. A., \& Baker, J. S. (2019). Reliability and validity of a novel futsal special performance test designed to measure skills and anaerobic performance. International Journal of Sports Physiology and Performance, 14(8), 1096-1102. https://doi.org/10.1123/ijspp.2018-0850

García-Angulo, A., \& García-Angulo, F. J. (2018). Análisis de los saques de esquina en relación con el rendimiento en la UEFA Euro fútbol sala 2016. Journal of Sport \& Health Research, 10(3), 403-414.

Giani, G., Soares, G. F., \& da Silva, S. A. (2018). Análise dos parâmetros técnico-tático dos gols da liga espanhola de fútbol sala 2015/2016. RBFF-Revista Brasileira de Fútbol sala e Futebol, 10(36), 69-76.

Giulano, F. M., de Oliveira Bueno, M. J., Marche, A. L., Nakamura, F. Y., Cunha, S. A., \& Moura, F. A. (2015). Characterization of the sprint and repeated-sprint sequences performed by professional futsal players, according to playing position, during official matches. Journal of Applied Biomechanics, 31(6), 423-429. https://doi.org/10.1123/jab.2014-0159

Gómez Ruano, M. Á., Méndez-Domínguez, C., Indaburu, A., \& Travassos, B. (2018). Goal effectiveness after players' dismissals in professional futsal teams. Journal of Sports Sciences, 37(8), 857-863. https:// doi.org/10.1080/02640414.2018.1531498

Gómez, M. Á., Moral, J., \& Lago, C. (2015). Multivariate analysis of ball possessions effectiveness in elite futsal. Journal of Sports Sciences, 33(20), 2173-2181. https://doi.org/10.1080/02640414.2015.10 75168

Guachi Flores, M. J., \& Cerón Ramírez, J. C. (2019). Diferencias biomecánicas del doble penal en fútbol sala entre deportistas de alto rendimiento y novatos. Lecturas: Educación Física y Deportes, 24(254), 24-32.

Hulka, K., \& Weisser, R. (2017). The influence of the number of players on workload during small-sided games among elite futsal players. Montenegrin Journal of Sports Science and Medicine, 6(1), 45-48.

Ibáñez Pérez, R., Huerta López, G., \& Meroño, A. J. (2020). Cuantificación de carga interna del entrenamiento en jugadores profesionales de fútbol sala. SPORT TK-Revista EuroAmericana de Ciencias del Deporte, 75-86. https://doi.org/10.6018/sportk.431161

Iedynak, G., Galamandjuk, L., Koryahin, V., Blavt, O., Mazur, V., Mysiv, V., \& Gurtova, T. (2019). Locomotor activities of professional futsal players during competitions. Journal of Physical Education and Sport, 19, 813-818.

Lago, C., Jiménez, A., Padrón, A., Mecías, M., \& Rey, E. (2020). Perceptions of the technical staff of professional teams regarding injury prevention in Spanish national futsal leagues: a cross-sectional study. PeerJ, 8, e8817. https://doi.org/10.7717/peerj.8817

Lago, C., Rey, E., Padrón, A., Prieto, J., \& Garcia, J. (2020). The relative age effect in professional futsal players. Journal of Human Kinetics, 72(1), 173-183. https://doi.org/10.2478/hukin-2019-0105

Méndez Domínguez, C., Gómez Ruano, M. A., \& Ruíz-Pérez, L. M. (2019). Influencia del momento crítico desfavorable en la aparición del 5vs4+p en fútbol sala de élite. Journal of Sports Training, 3(2), 3-11.

Méndez-Domínguez, C., Gómez-Ruano, M. A., Ruíz-Pérez, L. M., \& Travassos, B. (2019a). Goals scored and received in 5vs4 GK game strategy are constrained by critical moment and situational variables in elite futsal. Journal of Sports Sciences, 37(21), 2443-2451. https://doi. org/10.1080/02640414.2019.1640567

Méndez, C., Gómez, M. A., Ruíz, L. M., \& Travassos, B. (2019b). Goalkeeper as an outfield player: shooting chances at critical moments in elite futsal. International Journal of Performance Analysis in Sport, 19(2), 179-191. https://doi.org/10.1080/24748668.2019.1581967

Méndez, C., Travassos, B., Santos, J., Ribeiro, J. N., \& Gonçalves, B. (2019). Attacking profiles of the best ranked teams from elite futsal leagues. Frontiers in psychology, 10, 1370. https://doi.org/10.3389/ fpsyg.2019.01370
Miloski, B., de Freitas, V. H., Nakamura, F. Y., de Andrade Nogueira, F. C., Bara-Filho, M. (2016). Physical fitness, muscle damage and hormonal status. Journal of Strength and Conditioning Research: The Research Journal of the NSCA, 30(6), 1525-1533. https://doi.org/10.1519/ JSC. 0000000000001270

Moore, R., Bullough, S., Goldsmith, S., \& Edmondson, L. (2014). A systematic review of futsal literature. American Journal of Sport Science and Medicine, 2(3), 108-116.

Nakamura, F. Y., Pereira, L. A., Cal Abad, C. C., Kobal, R., Kitamura, K., Roschel, H., \& Loturco, I. (2016a). Differences in physical performance between U-20 and senior top-level Brazilian futsal players. Journal of Sports Medicine and Physical Fitness, 56(11), 1289-1297.

Nakamura, F. Y., Pereira, L. A., Rabelo, F. N., Ramirez-Campillo, R., \& Loturco, I. (2016b). Faster futsal players perceive higher training loads and present greater decreases in sprinting speed during the preseason. Journal of Strength and Conditioning Research, 30(6), 1553-1562. https://doi.org/10.1519/JSC.0000000000001257

Nascimento Júnior, J. R. A. D., Granja, C. T. L., Silva, A. A. D., Fortes, L. D. S., Gonçalves, M. P., Oliveira, D. V. D., \& Fiorese, L. (2019). Association between basic psychological needs of the self-determination theory and perception of group cohesion among high-performance futsal athletes. Revista Brasileira de Cineantropometria \& Desempenho Humano, 21, e57369.

Nascimento Junior, J. R. A., Nickenig Vissoci, J. R. N., Codonhato, R., de Sousa Fortes, L., Vicentine de Oliveira, D., Pestillo Oliveira, L., Vieria do Nascimento, J., \& Fiorese, L. (2018). Effect of the coaches' leadership style perceived by athletes on team cohesion among elite Brazilian futsal players. Cuadernos de Psicología del Deporte, 18(3), 252-267.

Nascimento Junior, J.R.A., Vissoci, J.R.N., Lavallee, D., Codonhato, R., Do Nascimento, J. V., \& Vieira, L. F. (2017). The mediating role of basic needs satisfaction on the relationship of perfectionism traits and team cohesion among elite futsal athletes. International Journal Sport Psychology, 48, 591-609. https://doi.org/10.7352/IJSP 2017.48.591

Naser, N., \& Ali, A. (2016). A descriptive-comparative study of performance characteristics in futsal players of different levels. Journal of Sports Sciences, 34(18), 1707-1715. https://doi.org/10.1080/026404 14.2015.1134806

Nunes, R. F. H., Buzzachera, C. F., Almeida, F. A. M., Da Silva, J. F., Flores, L. J. F., \& Da Silva, S. G. (2016). Relationships between isokinetic muscle strength, measures of aerobic fitness, single sprint performance, and repeatedsprint ability in elite futsal players. Gazzetta $\mathrm{Me}$ dica Italiana Archivio per le Scienze Mediche, 175(5), 205-13.

Palazón, M. A., Ortega, E., \& García-Angulo, A., (2015). Análisis bibliométrico de la producción científica en el fútbol sala. SPORT TK-Revista EuroAmericana de Ciencias del Deporte, 4(2), 19-24. https://doi. org/10.6018/242901

Pascual Verdú, N., Álvarez Amorós, A., Carbonell Martínez, J. A., \& Pérez Turpin, J. A. (2019). Análisis de patrón gol en competición de fútbol sala: $1^{\text {a }}$ División y $2^{\text {a }}$ División B. Retos: Nuevas Tendencias en Educación Física, Deporte y Recreación, 35, 364-368.

Paz-Franco, A., Rey, E., \& Barcala-Furelos, R. (2017). Effects of 3 different resistance training frequencies on jump, sprint, and repeated sprint ability performances in professional futsal players. The Journal of Strength \& Conditioning Research, 31(12), 3343-3350. https://doi. org/10.1519/JSC.0000000000001766

Pelana, R., Maulana, A., Winata, B., Widiastuti, W., Sukur, A., Kuswahyudi, K., \& Hermawan, R. (2019). Effect of contrast water therapy on blood lactate concentration after high-intensity interval training in elite futsal players. Physiotherapy Quarterly, 27(3), 12-19. https://doi. org/10.5114/pq.2019.86463

Sánchez-Sánchez, J., Bishop, D., García-Unanue, J., Ubago-Guisado, E., Hernando, E., López-Fernández, J., ... \& Gallardo, L. (2018). Effect of a Repeated Sprint Ability test on the muscle contractile properties in elite futsal players. Scientific Reports, 8(1), 1-8. https://doi. org/10.1038/s41598-018-35345-z

Sarmento, H., Bradley, P., Anguera, M. T., Polido, T., Resende, R., \& Campaniço, J. (2016). Quantifying the offensive sequences that result in goals in elite futsal matches. Journal of Sports Sciences, 34(7), 621-629. https://doi.org/10.1080/02640414.2015.1066024 
Sarmento, H., Bradley, P., \& Travassos, B. (2015). The transition from match analysis to intervention: optimising the coaching process in elite futsal. International Journal of Performance Analysis in Sport, 15(2), 471-488. https://doi.org/10.1080/24748668.2015.11868807

Sezen-Balçikanli, G., \& Sezen, M. (2017). Professional sports and empathy: relationship between professional futsal players' tendency toward empathy and fouls. Physical Culture and Sport. Studies and Research, 73(1), 27-35. https://doi.org/10.1515/pcssr-2017-0003

Vieira, L. H. P., Doğramaci, S. N., Barbieri, R. A., Milioni, F., Moura, F. A., Andrade, V. L. D., \& Santiago, P. R. P. (2016). Preliminary results on organization on the court, physical and technical performance of Brazilian professional futsal players: comparison between friendly preseason and official match. Motriz: Revista de Educação Física, 22(2), 80-92. https://doi.org/10.1590/S1980-6574201600020011
Wilke, C. F., Ramos, G. P., Pacheco, D. A., Santos, W. H., Diniz, M. S., Gonçalves, G. G., Bouzas Marins, J. C., Penna Wanner, S., \& SilamiGarcia, E. (2016). Metabolic demand and internal training load in technical-tactical training sessions of professional futsal players. Journal of Strength and Conditioning Research, 30(8), 2330-2340. https:// doi.org/10.1519/JSC.0000000000001321

Wilke, C. F., Wanner, S. P., Santos, W. H., Penna, E. M., Ramos, G. P., Nakamura, F. Y., \& Duffield, R. (2020). Influence of faster and slower recovery-profile classifications, self-reported sleep, acute training load, and phase of the microcycle on perceived recovery in futsal players. International Journal of Sports Physiology and Performance, 1(aop), 1-6. https://doi.org/10.1123/ijspp.2019-0201 